\title{
Aerostructural Optimization of Nonplanar Lifting Surfaces
}

\author{
Peter W. Jansen* \\ University of Toronto, Toronto, Ontario M3H 5T6, Canada \\ Ruben E. Perez \\ Royal Military College of Canada, Kingston, Ontario K7K 7B4, Canada \\ and \\ Joaquim R. R. A. Martins \\ University of Michigan, Ann Arbor, Michigan 48109 \\ DOI: $10.2514 / 1.44727$
}

\begin{abstract}
Nonplanar lifting surfaces can lower the induced drag relative to planar surfaces by redistributing vorticity. Other sources of drag, such as viscous drag, as well as nonaerodynamic considerations, such as structural weight, also play an important role in assessing the overall efficiency of such lifting surfaces. In this paper we solve a series of problems to find optimal nonplanar lifting surfaces and to explain the various factors and tradeoffs at play. A panel method and an equivalent beam finite-element model are used to explore nonplanar lifting surfaces, while taking into account the coupling and design tradeoffs between aerodynamics and structures. Both single-discipline aerodynamic optimization and multidisciplinary aerostructural optimization problems are investigated. The design variables are chosen to give the lifting-surface arrangement as much freedom as possible. This is accomplished by allowing a number of wing segments to vary their area, taper, twist, sweep, span, and dihedral, with the constraint that they must not intersect each other. Because of the complexity of the resulting design space and the presence of multiple local minima, an augmented Lagrangian particle swarm optimizer is used to solve the optimization problems. When only aerodynamics are considered, closed lifting-surface configurations, such as the box wing and joined wing, are found to be optimal. When aerostructural optimization is performed, a winglet configuration is found to be optimal when the overall span is constrained, and a wing with a raked wingtip is optimal when there is no such constraint.
\end{abstract}

\section{Nomenclature}

$b \quad=$ projected wing semispan, $\mathrm{ft}$

$b_{i} \quad=$ span of lifting-surface segment $i, \mathrm{ft}$

$C_{D}=$ drag coefficient

$C_{L}=$ lift coefficient

$C_{L_{\text {cruise }}}=$ cruise lift coefficient

$C_{L_{\text {mvr }}}=$ maneuver lift coefficient

$C_{L_{\mathrm{stall}}}=$ reference stall lift coefficient

\section{reference stall lift coefficient}

airfoil viscous drag coefficient

airfoil lift coefficient

$=$ root chord, $\mathrm{ft}$

$=$ total drag, $\mathrm{lbf}$

$=$ projected wing height, $\mathrm{ft}$

$=i$ th lifting-surface segment

$=j$ th vortex panel or beam element

$=$ total lift, lbf

$=$ number of lifting-surface segments

$=$ local Reynolds number

$=$ spar wall thickness, in

$=$ cruise speed, $\mathrm{kts}$

$=$ fuel weight burned during cruise, $\mathrm{lb}$

$=$ initial cruise weight, $\mathrm{lb}$ thrust specific fuel consumption, lb/(lbf-h)

$=$ diameter of circular cross section of spar, in.

Received 2 April 2009; revision received 6 May 2010; accepted for publication 30 May 2010 . Copyright $\odot 2010$ by the authors. Published by the American Institute of Aeronautics and Astronautics, Inc., with permission. Copies of this paper may be made for personal or internal use, on condition that the copier pay the $\$ 10.00$ per-copy fee to the Copyright Clearance Center, Inc., 222 Rosewood Drive, Danvers, MA 01923; include the code 0021-8669/ 10 and $\$ 10.00$ in correspondence with the CCC.

${ }^{*}$ Graduate Student, Institute for Aerospace Studies. Student Member AIAA.

${ }^{\dagger}$ Assistant Professor, Department of Mechanical and Aerospace Engineering. Member AIAA.

${ }^{\ddagger}$ Associate Professor, Department of Aerospace Engineering. Senior Member AIAA.

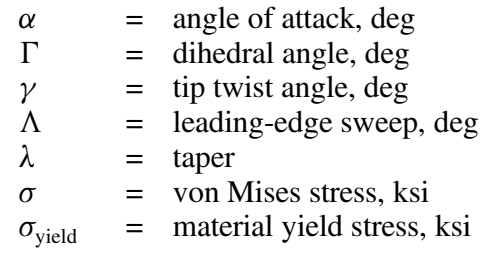

\section{Introduction}

$\mathbf{N}$ ONPLANAR lifting surfaces can offer significant gains in aerodynamic efficiency relative to conventional planar lifting surfaces for the same span and lift by lowering the total induced drag [1]. Numerous nonplanar wing concepts have been proposed. These concepts differ in the general arrangement of the lifting-surface geometries and the resulting aerodynamic characteristics.

Lift-induced drag is an important factor in the design of aircraft. For conventional airliners at the cruise condition, induced drag accounts for about $40 \%$ of the total drag. One way to reduce induced drag is to increase the wingspan of the aircraft. However, wing structural weight increases with the span, negating the reduced induced drag beyond a certain span. There can also be practical constraints on span imposed by the existing airport infrastructure.

One of the first nonplanar wingtip modifications was the addition of an end plate [2,3]. Whitcomb [4] called these end plates winglets and showed that they improve the lift-to-drag ratio when compared to wingtip extensions. The wing root bending moment of the two cases was matched in this study, but the wing was not redesigned.

Flechner and Jones [5] conducted studies on several transport aircraft configurations using the root bending moment to estimate structural weight. Their conclusion was that "the ratios of the relative aerodynamic gain over the relative structural weight penalty for winglets are 1.5 to 2.5 times those for wingtip extensions." On the other hand, based on a similar investigation, Jones and Lasinski [] concluded that the addition of a winglet does not provide a decisive advantage over a tapered wing extension. This was also the conclusion of Asai [7], who included viscous drag in his computational 
study, stating that tip extensions can provide a slight improvement over winglets. The reduction of induced drag by winglets is offset by the addition of wetted area (and hence parasitic drag) that does not contribute to lift.

Another type of nonplanar lifting-surface configuration is a multiplane arrangement. This includes vertically staggered wings (such as biplanes) and horizontally staggered wings (such as tandem wings). These configurations can exhibit lower induced drag, more laminar flow, and higher drag divergence Mach numbers [8]. However, considerations such as internal fuel volume, structural weight, and low Reynolds number effects have constrained their use in practice.

Closed configurations such as ring wings [9], box wings [10], and joined wings $[11,12]$ are other examples of nonplanar lifting-surface configurations. Closed lifting-surface arrangements aim to reduce the influence of the wingtip vortices. Among all nonplanar configurations, the box wing has been shown to achieve the minimum induced drag for a given lift, span, and vertical extent $[1,13]$. $\mathrm{C}$-wings, which can be seen as a compromise between a box-wing and a winglet configuration, provide a reduction in induced drag that approaches that of the closed configurations, without the viscous drag penalty incurred by a large wetted area [14].

Previous efforts in the analysis and optimization of nonplanar lifting surfaces have mostly focused on the aerodynamic aspects of these configurations. A systematic optimization of nonplanar lifting surfaces with the objective of minimizing induced drag was performed by Gage [15], which led to the discovery of the C-wing configuration.

Two more recent efforts focused on the tradeoff between tip extensions and winglets. The numerical study performed by Verstraeten and Slingerland [16] included viscous drag and root bending moment for structural considerations. They concluded that winglets only provide a benefit for wings with a span constraint and they did not find $\mathrm{C}$-wings to be advantageous.

Ning and Kroo [17] added area dependency to the weight calculation and highlighted the fact that the wing loading at the maneuver condition can be significantly different from the cruise condition wing loading, affecting the tradeoff between the aerodynamics and the structures. They also investigated the effect of viscous drag and stall constraints. They found that when wings were required to sustain higher maneuver loads, winglets performed slightly better than tip extensions. This study also addressed C-wings and concluded that they may have slightly lower drag compared to winglets, especially for designs with span constraints.

In spite of all these investigations, more work is needed in this area, since there is still no definite consensus on which wing configurations are truly optimal. The present work differs from previous efforts in three main aspects.

The first aspect is that this investigation gives as much freedom to the optimizer as possible. This is accomplished by representing the wing by a number of segments whose area, taper, twist, sweep, span, and dihedral can vary arbitrarily, subject only to a self-intersection constraint. The optimization with respect to such a wide range of design variables is made possible by the use of a gradient-free optimizer.

The second aspect is that aerostructural deflections are taken into account: Rather than optimizing the flying shape, we optimize the jig shape and compute the aerodynamic performance of the flying shape at cruise. The structure is sized based on a maneuver condition, which exhibits yet another wing shape.

Finally, the effect of structural sizing on the design optimization of the wing configurations is explored. Structural sizing affects the stiffness of the wing, and thus its flying shape, and its weight as well. This provides the optimizer with the means to exploit aerostructural tradeoffs by varying structural and aerodynamic design variables simultaneously.

The aerostructural model used in this work is described in the following section. Two main sets of results are then presented: a set of aerodynamic optimizations and a set of aerostructural optimizations. Within each of these sets, the problems differ in the set of design variables used, the components of drag considered, and whether span is constrained. The results of these investigations are summarized in the last section.

\section{Model Description}

While high-fidelity methods can provide accurate aerostructural analysis [18], limitations in the computational fluid dynamics volume grid generation prevent the optimization of general nonplanar shapes [19]. On the other hand, lower-fidelity models, such as aerodynamic panel methods, can quickly analyze arbitrary nonplanar configurations. Because of the wide range of allowable designs in the present work, the problem exhibits multiple local optima and thus a gradient-free optimization method is used.

\section{A. Aerodynamics}

The aerodynamic forces and moments are computed by a panel method that is based on potential flow theory and governed by Laplace's equation [20]. The lifting surfaces and the wake are discretized in the spanwise direction by vortex rings. The wake vortex rings close at the Trefftz plane. The panel method by itself can only compute the induced drag.

Viscous drag is a significant fraction of total drag, especially during the cruise segment. The main effect of considering viscous drag is that it adds a penalty to wetted area increases, particularly for surfaces that do not contribute to lift, such as winglets. Thus, considering viscous drag is particularly important for an optimization that allows the wing planform area to vary.

Several approaches can be used to extend the linear aerodynamic model to include viscous drag. One such approach consists in coupling the potential flow solver with a boundary-layer solver [20]. A much less computationally costly approach uses the drag polars for the airfoil and relates its section lift coefficients to the section drag coefficients [21]. It is assumed that the viscous drag coefficient of the airfoil varies quadratically with the section lift coefficient $[16,17]$, i.e.,

$$
c_{d}=c_{d_{0}}(R e)+c_{d_{1}}(R e) c_{l}+c_{d_{2}}(R e) c_{l}^{2}
$$

where the three coefficients in this equation depend on the airfoil characteristics and the local Reynolds number at the midspan of each aerodynamic panel. The coefficients are determined by interpolating the results for the airfoil at different Reynolds numbers and lift coefficients.

This approach is validated against wind tunnel tests for a wing with a NACA 65-210 airfoil. The wing geometry and test conditions are the same used by Sivellis [22]. The experimental and calculated drag polars are shown in Fig. 1. The agreement between the experimental and calculated results is sufficient for our purposes: The root-mean-

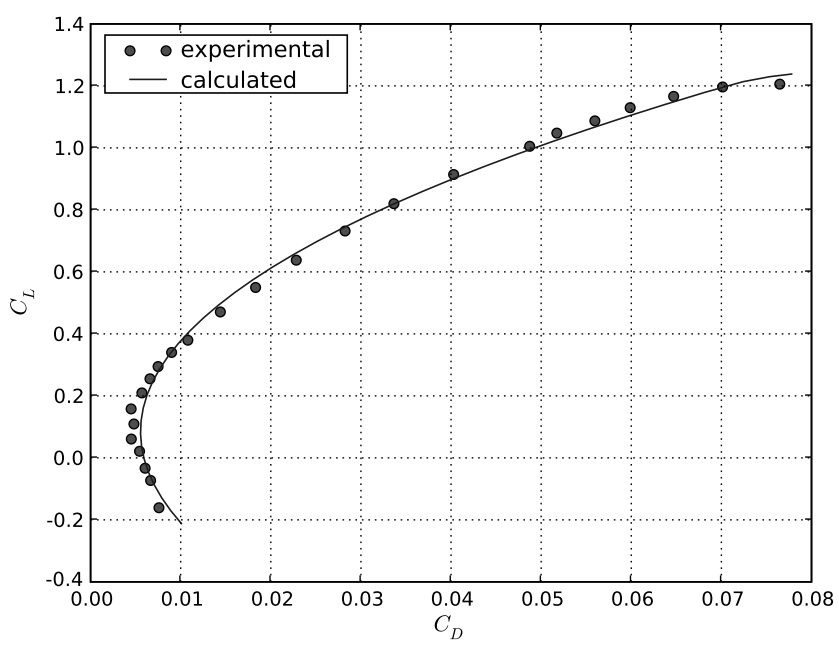

Fig. 1 Experimental and calculated drag polar. 
square error between the experimental data and our estimate is $1.2 \%$. At low lift coefficients, the approximation overpredicts the overall drag by up to $14.8 \%$. For such low lift coefficients, viscous drag is the dominant part of the total drag and the simple quadratic approximation cannot provide the required accuracy. Discrepancies also exist for the high lift-coefficient ranges, since flow separation is not predicted by the panel method.

\section{B. Structures}

The wing structure is modeled using an equivalent beam finite element approach. For lifting surfaces, equivalent beam representations have shown to adequately approximate stiffness and deflection behavior of the real wing $[23,24]$. As with the aerodynamic model, the computational cost of this structural model is modest, which is a requirement in the present work. The model uses space beam elements based on Euler-Bernoulli beam theory [25]. Each element has two nodes, each of which has 6 degrees of freedom: three displacements and three rotations. The spanwise discretization is based on the same intervals as those of the panel method discretization and thus the total number of vortex panels and beam elements $\left(n_{\text {elems }}\right)$ is the same. The centroidal axis of the structure can be specified to be at any chordwise location at a given segment connection. Figure 2 shows the geometry of the beam and the aerodynamic panels for a simple wing. The beam model represents a thin-walled circular section spar with diameter $d$ and wall thickness $t$.

\section{Aerostructural Analysis}

To obtain the aerostructural solutions, the aerodynamic analysis passes the external forces to the structural analysis, and the structures in turn passes the displaced shape of the wing back to the aerodynamic analysis. These force and displacement transfers are performed using a consistent and conservative scheme [26]. The system is converged using a fixed point iteration. Once the aerostructural analysis has converged, the wing drag and aircraft weight are passed to a module that computes the range. The stresses are computed with another aerostructural analysis at a maneuver condition. The range and element stresses are returned to the optimizer to determine the next design step.

\section{Optimizer}

The design space for nonplanar lifting surfaces is very complex and may include multiple local minima $[1,15]$. Therefore, a gradientfree optimizer that increases the probability of finding the global optimum is used. The optimizer is a parallel augmented Lagrange multiplier particle swarm optimizer (ALPSO), which enforces the constraints directly [27]. Particle swarm optimization (PSO) is inspired by the social behavior of insects in swarms, who adjust their flight paths to avoid predators, and to seek the best food sources [28,29]. The PSO algorithm is robust and well suited to handle nonlinear, nonconvex design spaces with discontinuities. Compared to other global design optimization methods, PSO has been shown to require fewer function evaluations and to yield results that are better or at least as good as the other methods [30-32]. Furthermore, for multimodal design spaces, the PSO algorithm is able to simultaneously find multiple local minima. The results for this paper are obtained by using the ALPSO algorithm run on an SGI Altix parallel computer equipped with Intel Itanium processors. To ensure that the

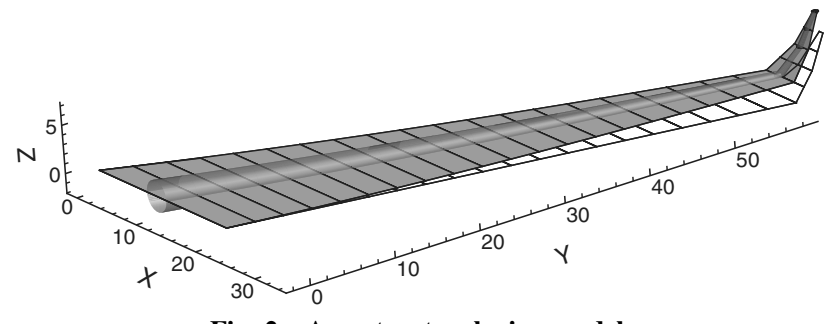

Fig. 2 Aerostructural wing model. solutions are local minima and to further refine the results, every problem is also solved using the gradient-based optimizer SNOPT [33] with the ALPSO result as the starting point.

\section{E. Test Case}

We chose to optimize the main lifting surface of a typical commercial aircraft at the cruise condition, with an added maneuver condition at which structural constraints are enforced. The specifications for this particular configuration are listed in Table 1 and are based on the Boeing 737-900 aircraft. A NACA 64A21 $\overline{2}$ airfoil was used for all problems. Note that the geometry constraints are only enforced in certain optimization problems. The various wing design optimization problems and corresponding results are presented in the following sections.

\section{Aerodynamic Optimization}

The purpose of solving the aerodynamic optimization problems that follow is to compare the results to what has been previously published, in order to validate our approach. In addition, these aerodynamic optimization results complement the aerostructural results presented in a later section by providing a motivation and by helping explain the final results.

\section{A. Design Variables}

The wing is represented by up to four segments. The geometry of each segment is defined by six design variables: span, area, taper, twist, sweep and dihedral. The segments must form a continuously connected wing, where the tip chord of each segment is the same as the root chord of the next segment. The discretization of the whole surface is performed automatically, for both the aerodynamic panel and beam models. Four possible geometries are shown in Fig. 3 to demonstrate the design freedom allowed in these optimization problems.

The leading edge of the first segment root is fixed at the global coordinate system's origin. The addition of new segments is not allowed during the optimization, since this would change the number of overall design variables during the optimization process and might result in a prohibitively large number of possible configurations.

Using a set of four segments can produce a wide variety of planform configurations, while keeping the number of design variables at a manageable level for a gradient-free method. The number of aerodynamic panels in each segment (which coincides with the number of structural finite elements) depends on the segment's contribution to the overall continuous span. This ensures that all aerodynamic panels and structural finite elements have a similar width, which is desired for these models.

\section{B. Geometric Constraints}

Some wing segment arrangements are problematic to solve using a panel method. If two panels intersect or a vortex line lies too close to a control point, the aerodynamic influence coefficient matrix becomes ill-conditioned. In addition, when two panels lie on top of each other (i.e., the relative dihedral between two consecutive panels is $180 \mathrm{deg}$ ), the linear system of equations no longer has a unique solution.

These limitations require the introduction of additional constraints on the planform geometry. Before performing the aerodynamic analysis for a given design point, the planform geometry is checked to ensure that wing segments do not intersect or lie on top of each other and that vortex lines are not too close to a control point.

Designs that fail any of these checks cannot be analyzed using the panel code and are thus considered infeasible. Such designs are rejected by the ALPSO algorithm. However, the optimizer continues updating the swarm information toward feasible designs.

Without geometric or structural constraints, the span tends to increase indefinitely. If the span is constrained, nothing prevents the height from increasing indefinitely as well. To ensure that the aerodynamic optimization problems are well posed, the projected 

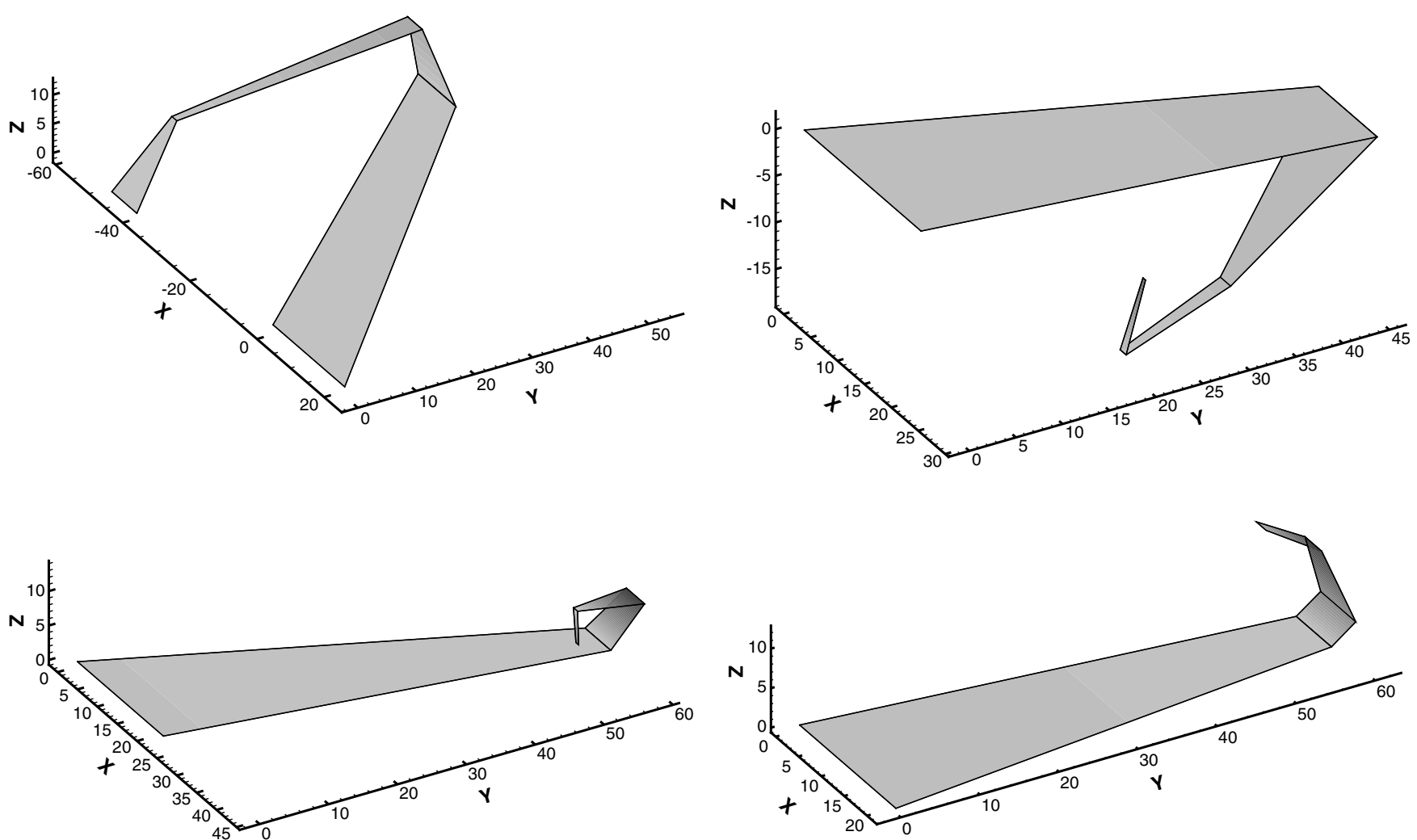

Fig. 3 Sample of possible wing geometries.

span and height are constrained to lie within a bounding box by specifying a maximum span and a maximum height.

\section{Induced Drag}

The first optimization problem is the aerodynamic optimization of rectangular lifting-surface segments for which only lift-induced drag is considered. The optimization problem is formulated as follows:

$$
\begin{aligned}
& \min _{\alpha, b_{i}, \Gamma_{i}, \gamma_{i}} D_{\text {induced }} \\
& \text { subject to }\left\{\begin{array}{l}
L=L_{\text {target }} \\
b \leq 60 \\
h \leq 12 \\
-15 \leq \alpha \leq 15 \\
0 \leq \Gamma_{i} \leq 190 \\
-15 \leq \gamma_{i} \leq 15
\end{array}\right.
\end{aligned}
$$

where $i=1, \ldots, n_{\text {segs }}$. The twist distribution varies linearly from the root to the tip for each lifting-surface segment. The lift constraint ensures that the wing matches the required total lift. The projected span and height constraints ensure that the configuration stays within a bounding box.

Figure $\underline{4}$ shows the optimal configuration for rectangular lifting surfaces obtained by solving this problem. The optimal configuration is a closed box wing with maximum span and height. This is the same result as that predicted by lifting-line theory. The span reduces the induced drag, and so does the height, by reducing the downwash induced by the two planar wing segments on each other. The lift distribution for the obtained wing is shown in Fig. 5a. Both planar wing segments carry approximately the same lift, $\overline{\text { and }}$ the vertical segment connecting the two planar segments does not contribute to lift. The elliptical lift distribution for the same total lift is also shown. The induced drag of this configuration is $24 \%$ lower than an optimal planar reference wing. This reference wing was obtained by solving the optimization problem (2) with the segment dihedrals fixed to zero. The twist distribution for the lifting-surface segments is shown in Fig. 5 b.

\section{Compressibility Drag}

The optimization problem described above uses only rectangular lifting surfaces and allows the optimizer to change only the span and
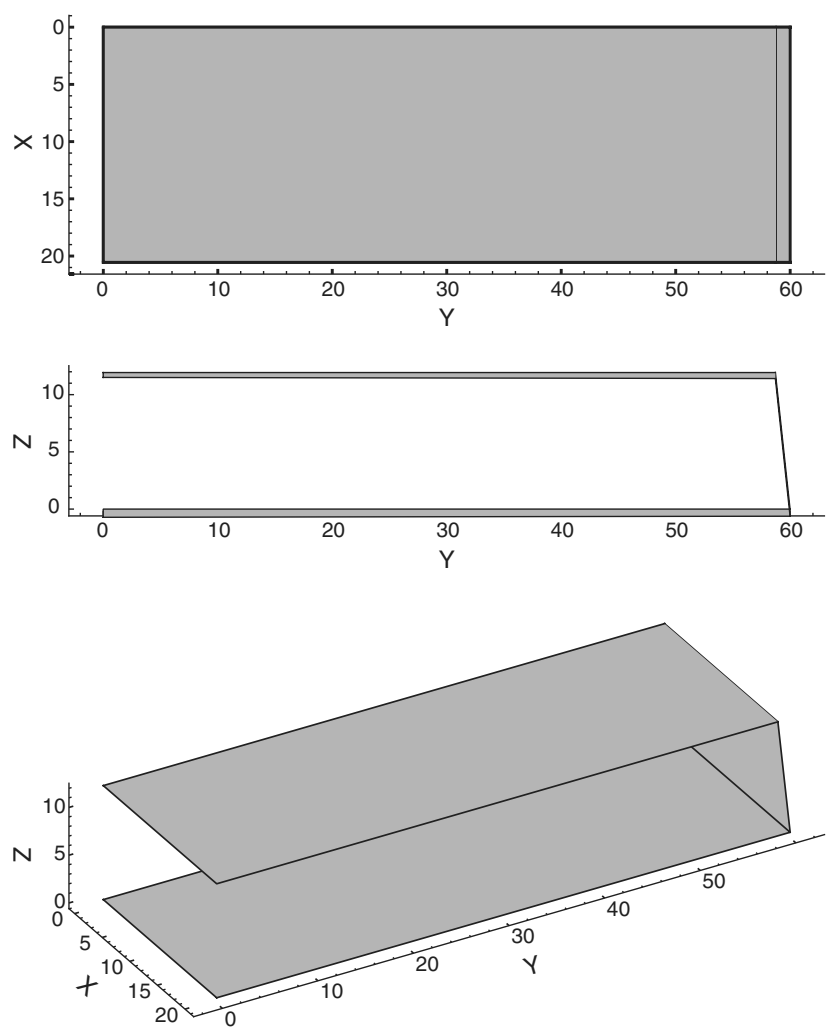

Fig. 4 Minimum-induced-drag solution. 


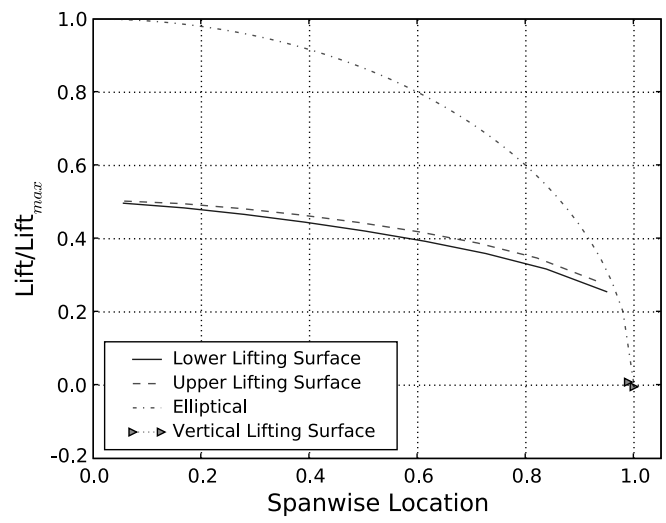

a) Lift distribution

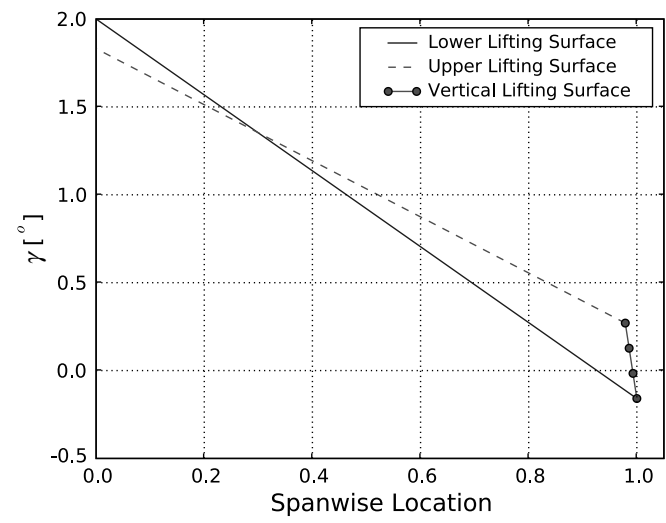

b) Twist distribution

Fig. 5 Spanwise distributions for minimum induced drag.

dihedral of each lifting-surface segment, but not the overall planform geometry. To obtain more practical planform geometries for the lifting surfaces that can be used for typical jet airliners, drag due to compressibility effects is added to the drag computation.

The sweep and taper of each segment are now included as design variables. Sweep plays a major role in reducing the wave drag. Including taper as a design variable can lead to very small tip chords. These small tip chords can result in high local lift coefficients that exceed the maximum section lift coefficients. Thus, a lift-coefficient constraint is added to prevent stall. The new optimization problem is as follows:

$$
\begin{aligned}
& \min _{\alpha, b_{i}, \Lambda_{i}, \Gamma_{i}, \gamma_{i}, \lambda_{i},} D_{\text {induced }}+D_{\text {compressibility }} \\
& \text { subject to }\left\{\begin{array}{l}
L=L_{\text {target }} \\
\left.c_{l}\right|_{C_{L_{\text {stall }}} \leq c_{l_{\max }}} \\
b \leq 60 \\
h \leq 12 \\
-15 \leq \alpha \leq 15 \\
0 \leq \Gamma_{i} \leq 190 \\
-15 \leq \gamma_{i} \leq 15 \\
-60 \leq \Lambda_{i} \leq 60 \\
0.05 \leq \lambda_{i} \leq 5
\end{array}\right.
\end{aligned}
$$

To impose section lift-coefficient constraints, the $c_{l}$ is evaluated for each panel at the condition corresponding to a reference stall lift coefficient $C_{L_{\text {stall }}}$ and constrained to be lower than or equal to a maximum lift coefficient of the airfoil, $c_{l_{\max }}$. If these constraints are not active, then the wing can produce more lift without stalling.

The root chord of the wing is fixed throughout the optimization. Large chords reduce the section lift coefficient and the local drag coefficient. If the optimizer were allowed to vary the root chord of the first lifting-surface segment, then this would result in unreasonably large chords, since there is no penalty for wetted area or structural weight in this problem. Both sweep and taper are bounded, to keep them within reasonable ranges. Note that taper is allowed to be greater than one.

As shown in Fig. 6, the solution of the optimization problem (3) is a closed joined wing. By sweeping the top and bottom wings in opposite directions, both compressibility drag and interference effects between the lifting surfaces are reduced. The vertical segment is again at the maximum height enforced by the geometric constraint. The segments have a taper ratio of one. Since no structural analysis is included in this analysis, there is no benefit in reducing the tip load. The drag of this configuration is $26 \%$ lower than the planar reference wing, which is obtained by solving the optimization problem (3) with the segment dihedrals fixed to zero.
Figure 7a shows the spanwise lift distribution for the optimal configuration at the cruise lift coefficient and the reference stall lift coefficient. The lower surface carries the most of the lift, while the upper surface carries a smaller fraction. The $c_{l}$ distribution is flat for both segments and is below the maximum section lift coefficient
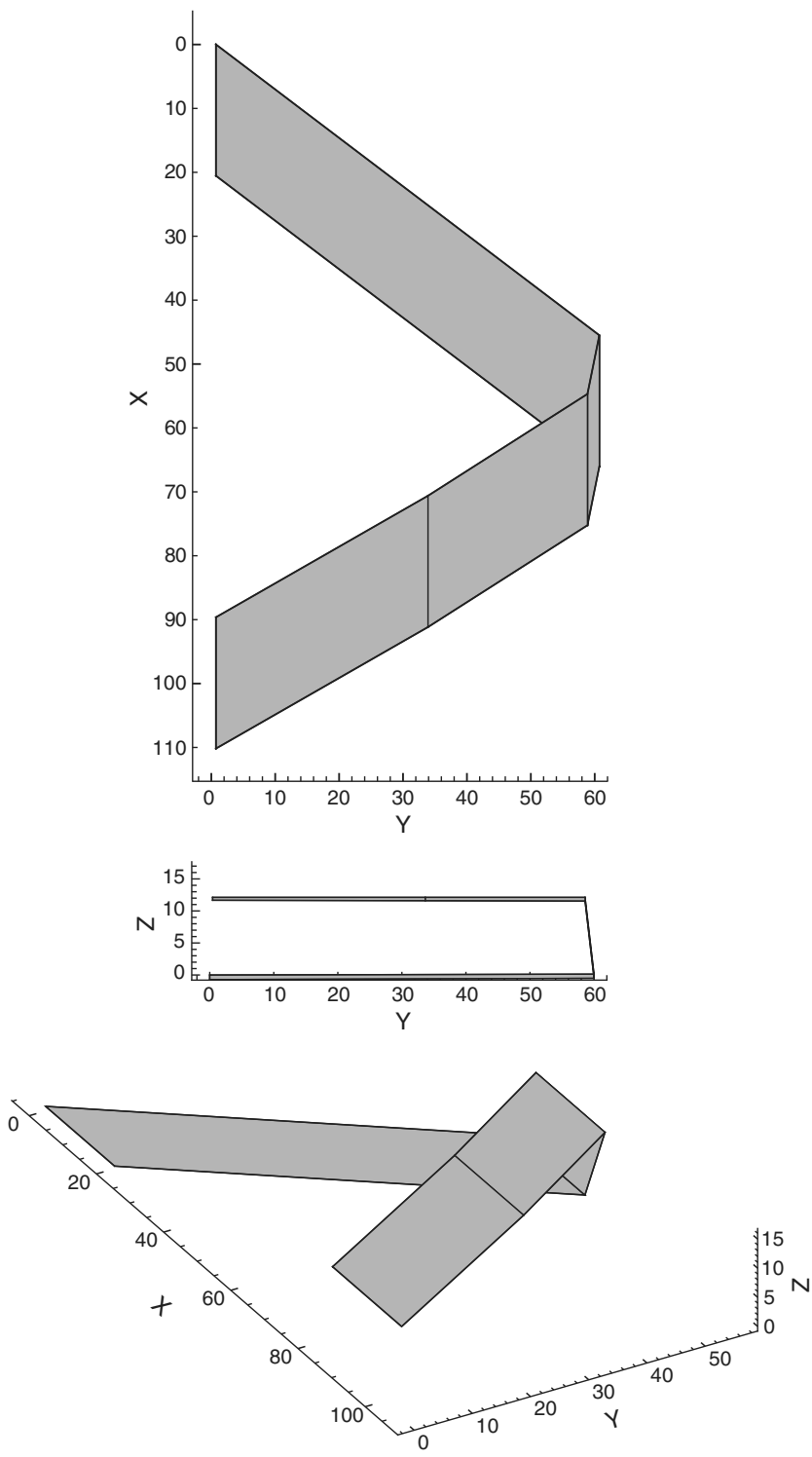

Fig. 6 Minimum-drag solution including induced and compressibility drag. 


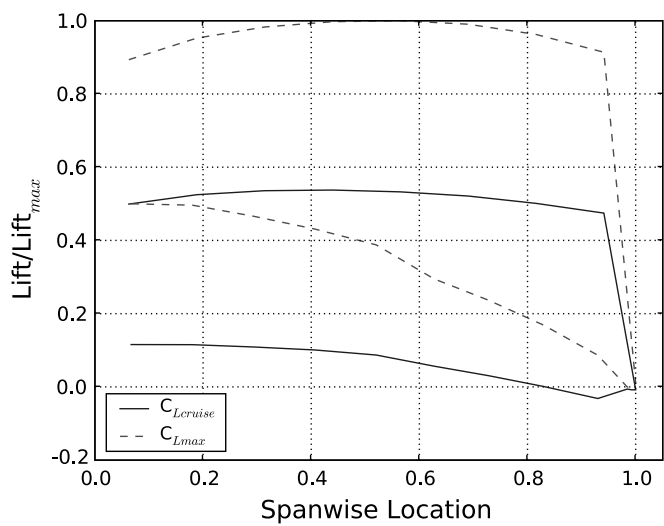

a) Lift distribution

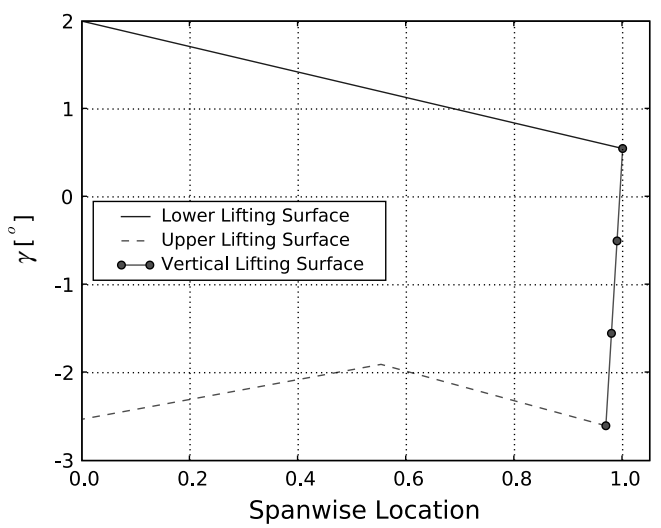

b) Twist distribution

Fig. 7 Spanwise distributions for minimum-drag solution, including induced and compressibility drag.

constraint. The twist distribution for the lifting-surface segments is shown in Fig. $7 \mathrm{~b}$.

\section{E. Viscous Drag}

The optimizations performed so far have considered only induced and compressibility drag. While including viscous drag has usually only a small effect on the optimal loading on a planar wing, it has a pronounced impact when considering nonplanar lifting surfaces. In this case, one can add surface area that does not contribute to lift but decreases induced drag.

The viscous drag is computed by using airfoil data, as explained in Sec. II.A, and then added to the total drag. The optimization problem is the same as the previous problem (3), except for the addition of this new source of drag and one additional design variable. Since there is now a penalty for a surface area increase, the root chord of the first segment, $c_{r}$, is included as a design variable.

The solution of this problem is the $\mathrm{C}$-wing shown in Fig. $\underline{8}$. All segments are tapered, which reduces the viscous drag for a given span. An inspection of the $c_{l}$ distribution at cruise revealed that for some of the panels around two-thirds of the semispan, the stall constraint is active. The remaining outboard sections of the main wing are close to the maximum section lift coefficient.

The spanwise lift distribution for the C-wing is shown in Fig. 9a. The top horizontal lifting surface carries a small amount of $\overline{\text { lift }}$ relative to the main wing. Since the viscous drag is dependent on the section lift coefficient, this results in low viscous drag from this segment.

The area of nonplanar segments now carries a viscous drag penalty, which needs to be offset by a reduction in induced drag. The $\mathrm{C}$-wing configuration provides an induced drag reduction similar to that of a box-wing or joined-wing configuration, but significantly reduces the wetted area of the wing. The root chord is $4 \%$ larger than the fixed root chord of the previous cases. The larger chords and the taper of the wing segments can be explained by considering the factors affecting viscous drag. A larger chord can reduce the drag, since larger Reynolds numbers result in smaller friction drag coefficients. The viscous drag coefficient also increases quadratically with the section lift coefficient, which is decreased when the chord increases for a given section lift. On the other hand, the viscous drag increases with wetted area. These effects work against each other, resulting in a particular optimal chord distribution and total wetted area.

The C-wing configuration achieves a drag reduction of $22 \%$ relative to the optimal planar reference wing. The twist distribution for the lifting-surface segments is shown in Fig. 7b. A local minimum corresponding to a wing with a winglet is also found when starting from a different set of design points. The drag of the $\mathrm{C}$-wing is $5 \%$ lower than the winglet local minimum.

Table $\underline{2}$ summarizes the results for the three different aerodynamic optimization problems. The drag coefficient values are calculated with respect to the fixed reference area for all cases. As previously explained, the planar wing configuration reference values for each case are obtained by solving the respective problem with the additional restriction that the dihedral for all segments be zero, which results in a planar wing in the $x-y$ plane.

\section{Aerostructural Optimization}

The aerodynamic optimization results presented in the previous section are not representative of actual wing design, because the effect of the spanwise lift distribution on the wing structural weight is not considered. By modeling the wing structure and optimizing with respect to structural sizing, we can perform interdisciplinary tradeoffs that are representative. In addition, it is more appropriate to
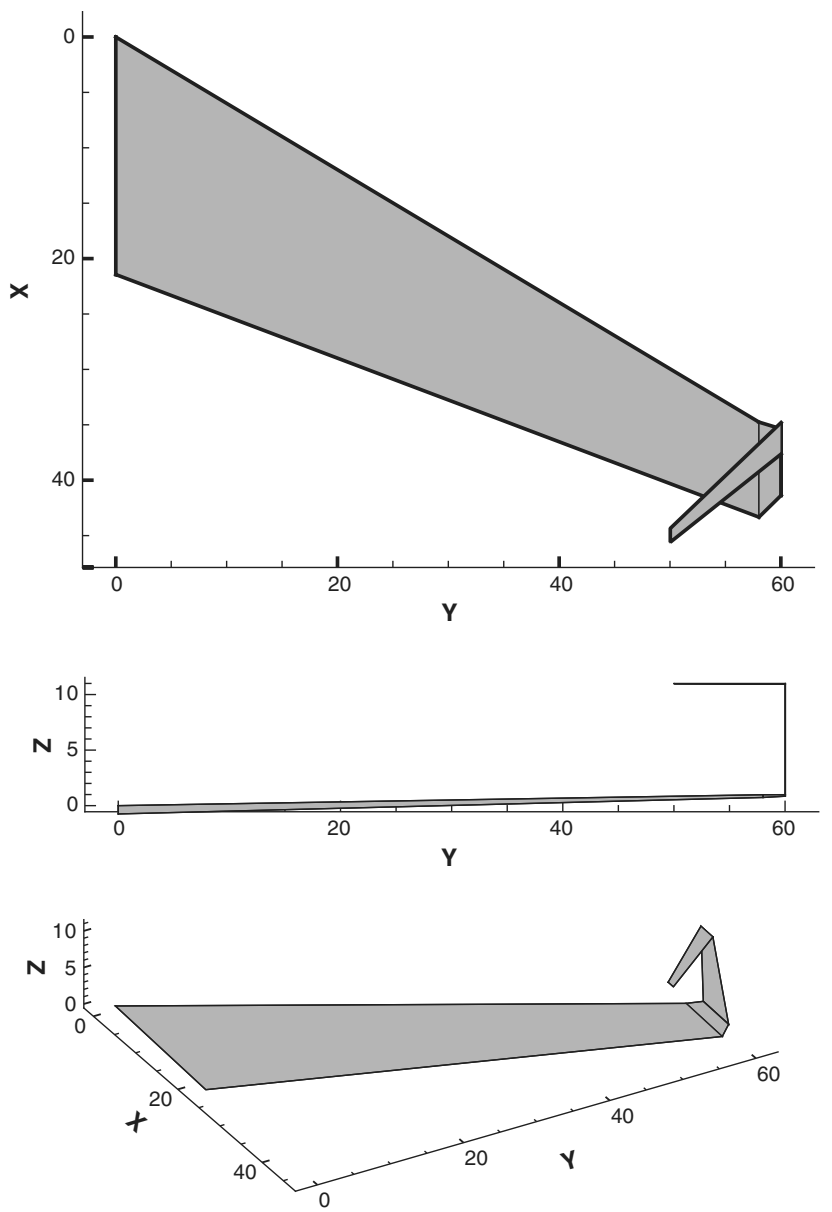

Fig. 8 Minimum-drag solution including induced, compressibility, and viscous drag. 


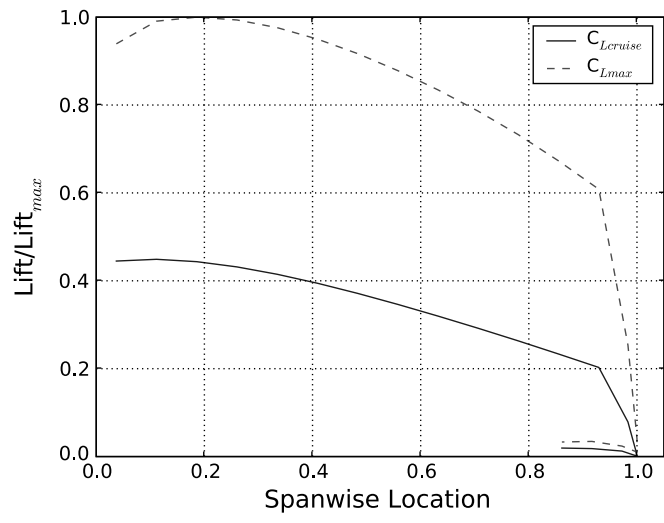

a) Lift distribution

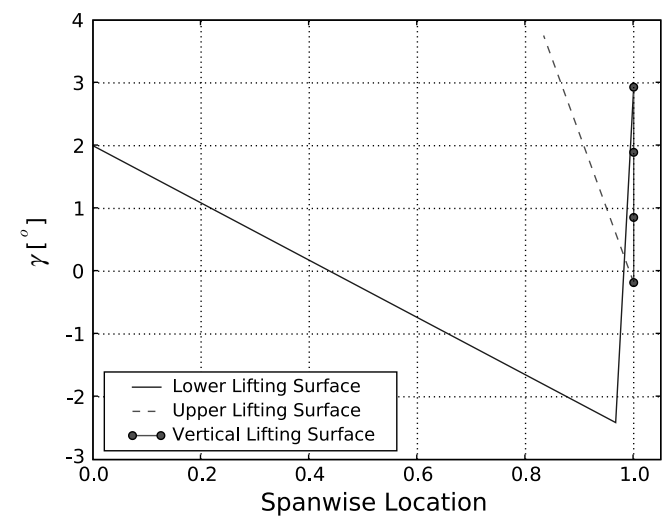

b) Twist distribution

Fig. 9 Spanwise distributions for minimum-drag solution, including induced, compressibility, and viscous drag.

optimize the jig shape, as opposed to optimizing the deflected shape and assuming that a structure can be designed to exhibit that exact shape for a fixed set of aerodynamic loads.

A suitable objective function can also play a major role in the interdisciplinary tradeoffs. The Breguet range equation is one of the simplest objective functions that can accomplish this, as it includes both aerodynamic and structural performance metrics. The range is thus computed as follows:

$$
\text { range }=\frac{V}{c} \frac{L}{D} \ln \left(\frac{W_{\text {initial }}}{W_{\text {initial }}-W_{\text {fuel }}}\right)
$$

The initial weight of the aircraft, $W_{\text {initial }}$, consists of the aircraft weight plus structural weight of the wing and a fixed fuel weight. A constant viscous drag component is added to the drag computed from the aerodynamic model to account for the drag of the fuselage and empennage.

The aerostructural optimization problem is similar to the aerodynamic optimization, with the only difference being the addition of structural considerations. As in the case of the aerodynamic optimization, the geometry is represented by up to four continuous segments defined by the previously described design variables. The aerostructural optimization problem is as follows:

$$
\begin{aligned}
& \max _{\alpha, c_{r}, b_{i}, \Lambda_{i}, \Gamma_{i}, \gamma_{i}, \lambda_{i}, t_{j}} \text { range } \\
& \text { subject to }\left\{\begin{array}{l}
L=W_{\text {initial }} \\
\frac{C_{L_{\mathrm{mvr}}}}{C_{L_{\mathrm{cruise}}}}=2.5 \\
\left.\left(c_{l}\right)_{j}\right|_{C_{L_{\mathrm{mvr}}} \leq c_{l_{\mathrm{max}}}} \\
\left.\sigma_{j}\right|_{C_{L_{\mathrm{mvr}}} \leq \sigma_{\text {yield }}} \\
-15 \leq \alpha \leq 15 \\
0 \leq \Gamma_{i} \leq 190 \\
-15 \leq \gamma_{i} \leq 15 \\
-60 \leq \Lambda_{i} \leq 60 \\
0.05 \leq \lambda_{i} \leq 5 \\
0.06 \leq t_{j} \leq \frac{1}{2} d_{j}
\end{array}\right.
\end{aligned}
$$

where for each beam finite element we add the thicknesses of the spar wall as design variables $\left(t_{j}\right.$, for $\left.j=1, \ldots, n_{\text {elems }}\right)$. The wall thicknesses must not exceed the spar radius and must be larger than the specified minimum gauge (0.06 in.). The lift is constrained to match the initial weight of the full aircraft, which is now a function of the structural thicknesses. The actual total wing weight is 1.85 times the weight obtained from the equivalent beam, where the diameter of each equivalent beam element is set to $16 \%$ of the local panel midchord. This weight multiplier has been found to give a good prediction for the wing weight of different transport aircraft [34].

To ensure structural integrity, element stress constraints are also added to the optimization problem. The stresses are calculated for a steady $2.5 \mathrm{~g}$ maneuver condition with a 1.5 safety factor as required for transport aircraft by Federal Aviation Regulations (FAR) Part 25 [35]. The wing optimization problem becomes even more interesting when the structural stress constraints are enforced at the maneuver condition. This is due to the fact that the spanwise lift distribution at the cruise condition can be shaped differently from the one at the maneuver condition. The ideal lift distribution at cruise is elliptical for minimum induced drag. On the other hand, the ideal lift distribution for minimum weight is one that shifts the lift inboard, which reduces the internal bending moments. This is desired at the maneuver condition, for which the structure is sized. The change in lift distribution between these two conditions can be tailored with the appropriate selection of taper and twist variables.

Including structural considerations in the optimization enables us to remove span and height constraints without running the risk of posing an ill-defined optimization problem. The span constraint is no longer necessary, because an increase in span dramatically increases the bending moment, requiring a much heavier structure to withstand the maneuver condition. Large vertical segments also incur a significant structural weight penalty. Constraints on the wing geometry might still be necessary due to other considerations, such as the gate constraint for certain airports.

The aerostructural optimization problem (5) is solved using the multidisciplinary feasible approach [36]. This is a single-level formulation that requires the solution of the multidisciplinary analysis at each optimization iteration. In the case of the aerostructural problem, this consists of an aerostructural solution that is obtained by solving the coupled system of aerodynamic and structural governing equations. The result is the flying wing shape for the given set of design variables and flight conditions.

At each optimization iteration, the objective function and constraints corresponding to the aerostructural solution are returned to the optimizer. Note that two aerostructural solutions are required: one at $C_{L_{\text {cruise }}}$ and another at $C_{L_{\text {mvr }}}$. The former yields the range (the

Table 2 Aerodynamic optimization results summary

\begin{tabular}{lcccr}
\hline \hline Types of drag considered & Segment geometry & Planar $C_{D}$ & Nonplanar $C_{D}$ & Ratio \\
\hline Induced & Rectangular & 0.01566 & 0.01191 & 0.76 \\
Induced, compressibility & Sweep and taper & 0.01364 & 0.01006 & 0.74 \\
Induced, compressibility, viscous & Sweep and taper & 0.01878 & 0.01456 & 0.78 \\
\hline \hline
\end{tabular}



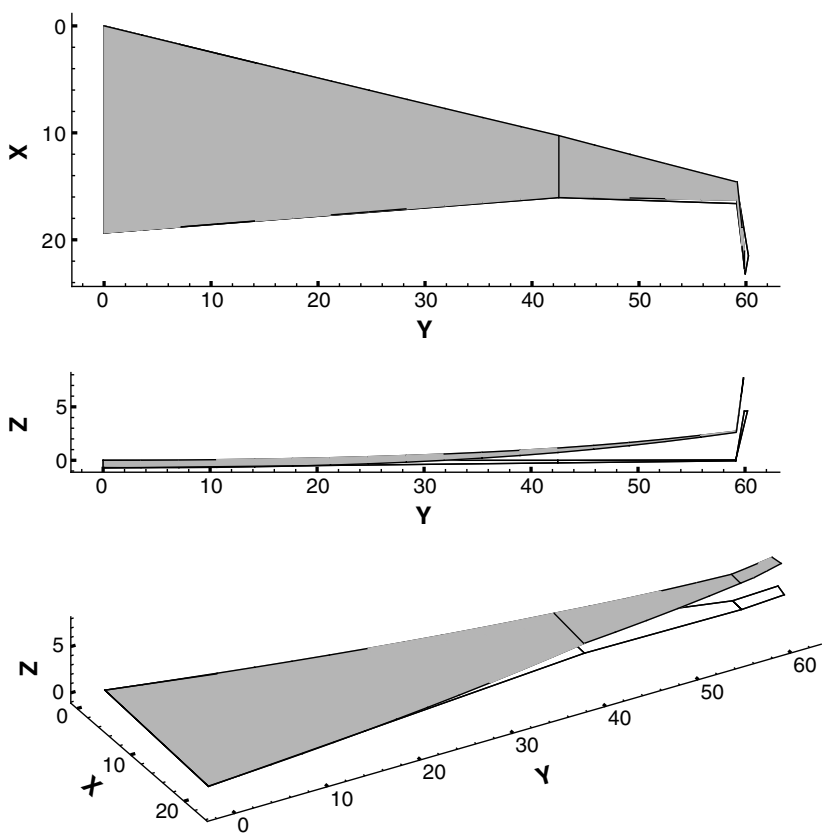

Fig. 10 Maximum-range solution with span constraint, including induced drag.

objective function), and the latter is used to compute the structural stress constraints.

\section{A. Constrained Span}

As mentioned above, a span constraint is no longer required to avoid an ill-defined optimization problem. However, it may be necessary to impose a span constraint due to airport operational requirements. Thus, we solve the aerostructural optimization problem (5) with the projected wingspan constraint, $b \leq 60$.

\section{Induced Drag}

This problem is first solved by considering only induced drag in the aerodynamic analysis. The optimal solution found by the optimizer is a wing configuration with a winglet, as shown in Fig. 10. The main lifting surface consists of two lifting-surface segments, while the winglet is represented by a single segment. The fourth segment was removed by the optimizer. The winglet is swept and has a dihedral angle of $76^{\circ}$. Since the projected span is constrained, this results in a smaller span for the planar segment than would be possible for a $90^{\circ}$ winglet.

The load distribution, shown in Fig. 11a, is closer to elliptical at the cruise condition, which reduces the induced drag. At the maneuver condition, the lift is shifted inboard, which alleviates the bending moment at the critical flight condition. This reduces the structural weight of the main segment and offsets the weight incurred by adding the winglet.

The taper of the lifting surfaces affects the diameters of the beam elements, which are dependent on the local chord. For a tapered segment the beam is thicker at the root for the same area and can resist a higher root bending moment. The sweep of the wing segments results in a reduced loading of the tip and the lift distribution is shifted inboard, due to the negative twist of the swept beam at higher loading conditions.

The thickness of the beam elements decreases from the root to the tip, resulting in fully stressed elements, as shown in Figs. 11c and $11 \mathrm{~d}$, respectively. Only the tip element of the winglet reaches the minimum gauge thickness. The twist distribution is shown in Fig. $11 \mathrm{~b}$ for the jig shape, as well as for the 1 and $2.5 \mathrm{~g}$ flight conditions. Because of sweep, the wing twist decreases with increased loading.

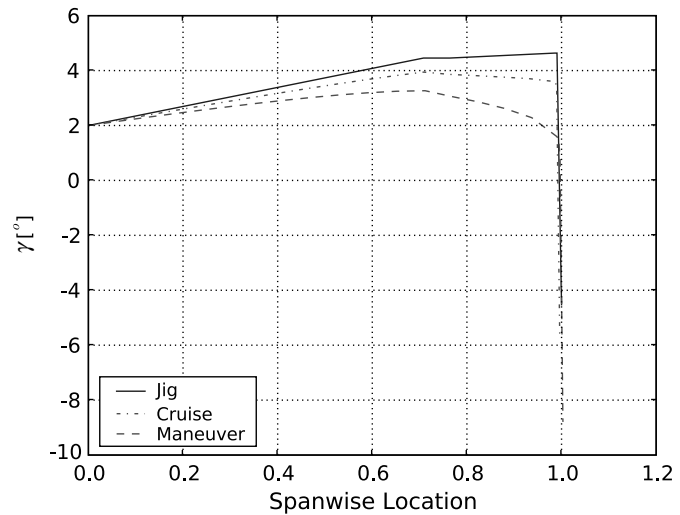

b) Twist distribution

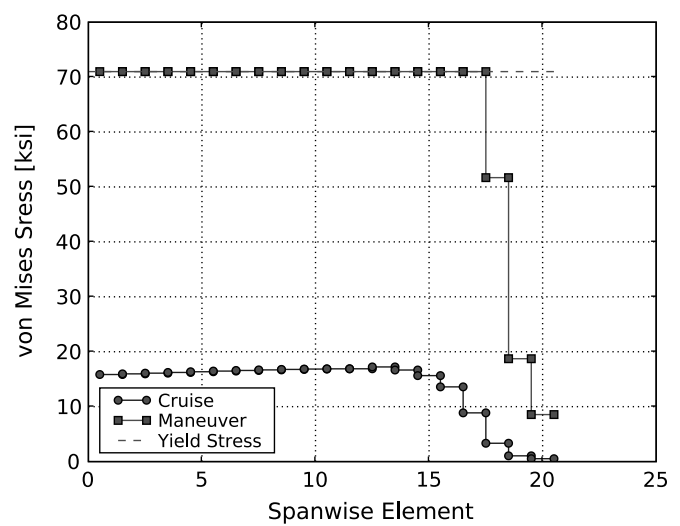

d) Stress distribution

c) Thickness distribution

Fig. 11 Spanwise distributions for span-constrained maximum range, including induced drag. 

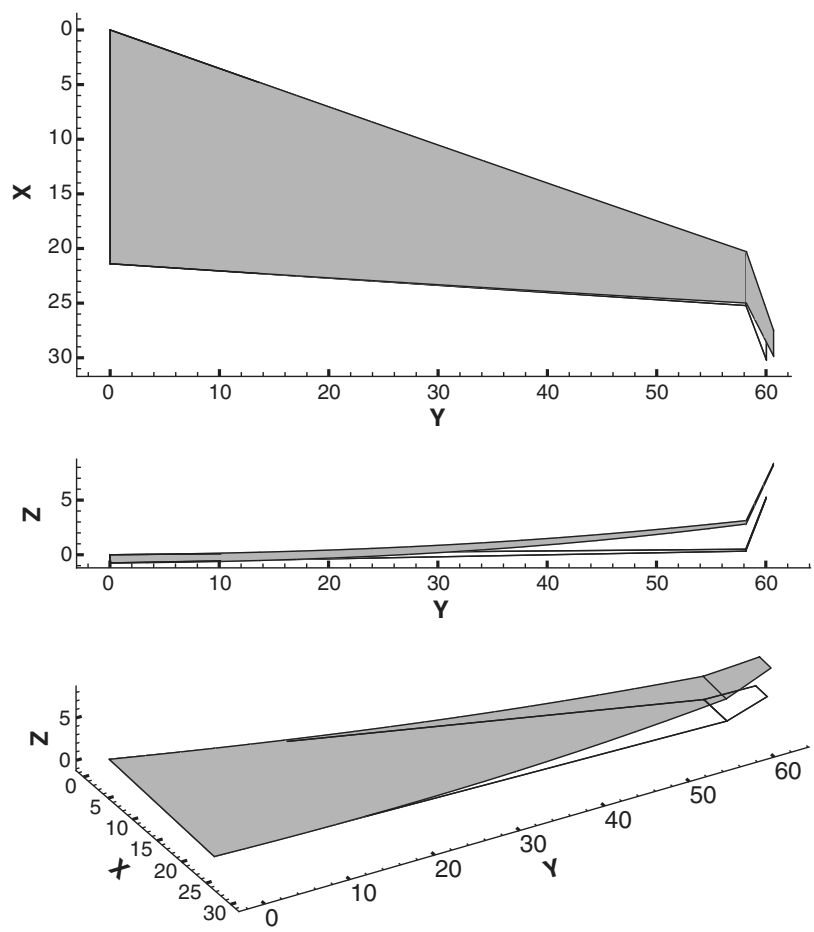

Fig. 12 Maximum-range solution with span constraint, including induced, compressibility, and viscous drag.

\section{Viscous and Compressibility Drag}

Viscous and compressibility drag are now included. Adding viscous drag decreases the sensitivity of the aircraft performance to changes in induced drag. Hence, a higher reduction in induced drag is required to offset the same increase in structural weight, assuming the viscous drag can be kept constant. The optimal solution is again a winglet configuration, as shown in Fig. 12. The height of the winglet is $30 \%$ larger when compared to the previous case.

The lift distribution shown in Fig. 13a exhibits a similar behavior to the previous case. Again, the load is shifted toward the root at the maneuver condition to reduce the bending moment distribution. Since the loading on the winglet is low, its structural thickness is driven to the minimum gauge value.

The root chord of this configuration is $10 \%$ larger than in the previous case, for which only induced drag is considered. Comparing the wing planform shape as a whole, the wing chords are larger, since they simultaneously increase the Reynolds number (hence decreasing the friction drag coefficient) and decrease the section lift coefficients (which also decrease the viscous drag). On the other hand, it does not pay off to increase the chord indefinitely, since the increase in viscous drag due to increased wetted area eventually catches up with these effects. At the same time, an increase in chord results in an increase in the spar diameter, which allows for a thinner wall, but beyond a certain diameter, an increase in structural weight is inevitable.

In Sec. III we found that the aerodynamic minimum-drag solutions, which were span-constrained, were a box wing when only induced drag is considered and a C-wing when viscous drag was added. The results in this section show that when structural considerations are added to the span-constrained optimization problem, the solutions for maximum range are winglet configurations. Winglet configurations provide lower drag than the planar configurations of the same span without adding substantial weight to the wing structure. The box-wing and the C-wing configurations, on the other hand, add more weight than their respective drag reductions can compensate for. Thus, a winglet configuration provides the best tradeoff between aerodynamics and structures when span is constrained.

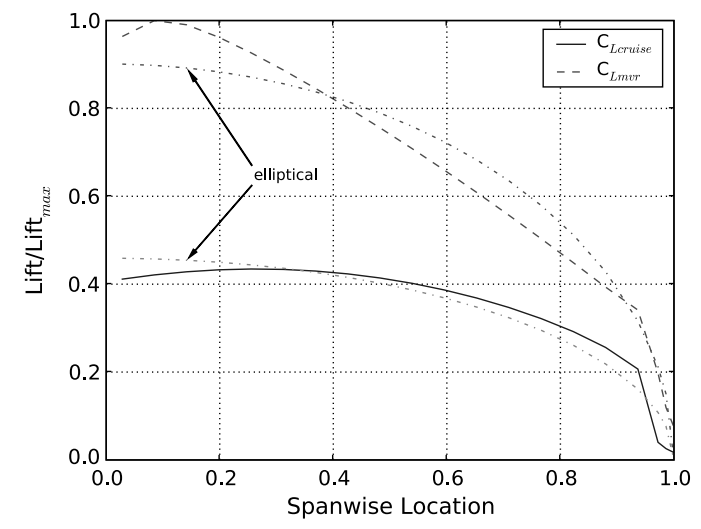

a) Lift distribution

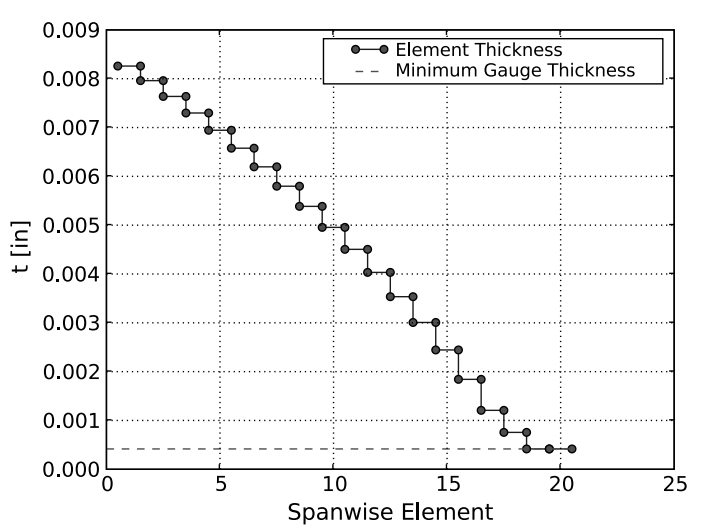

c) Thickness distribution

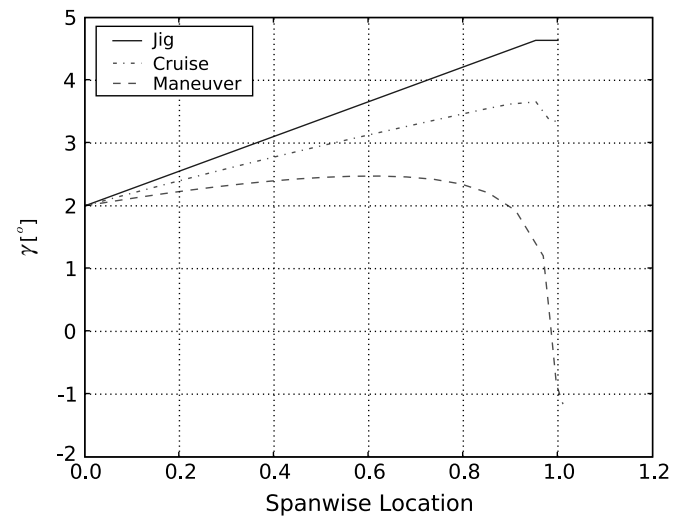

b) Twist distribution

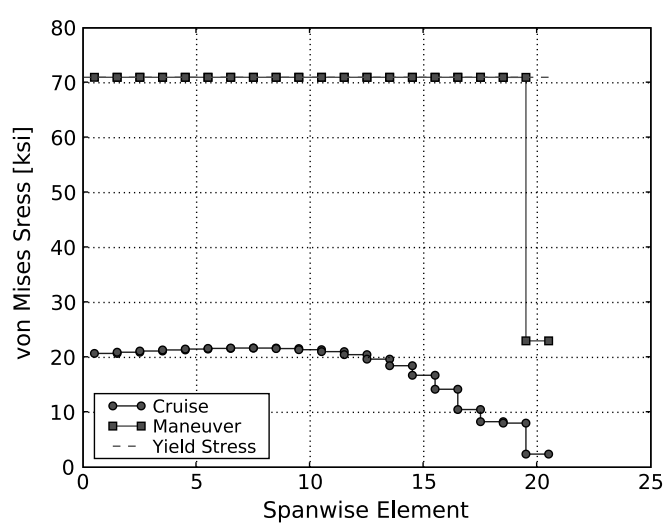

d) Stress distribution

Fig. 13 Spanwise distributions for span-constrained maximum range, including induced, compressibility, and viscous drag. 

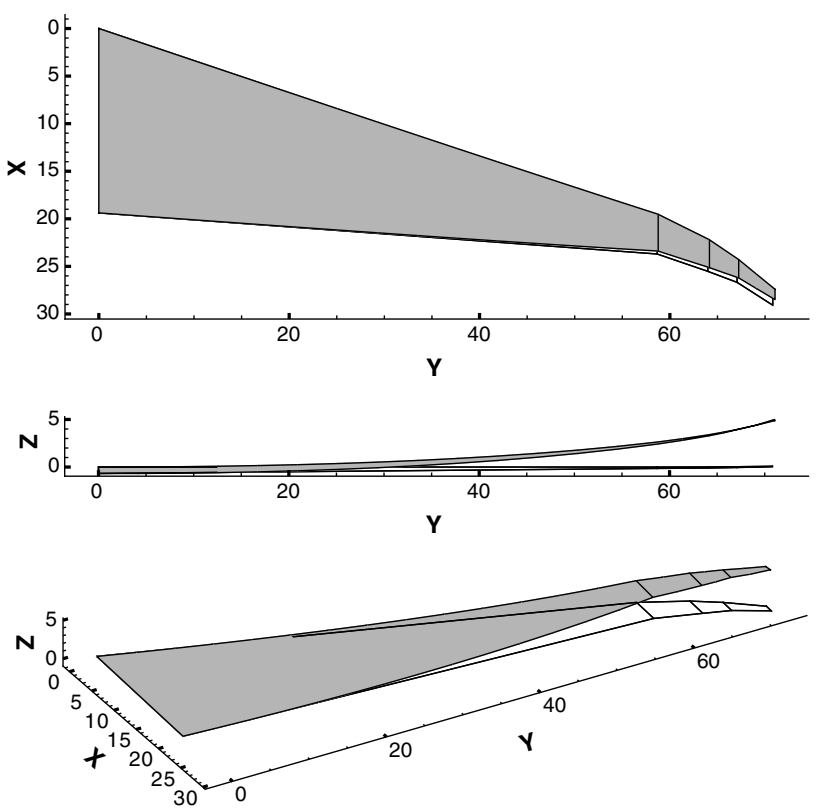

Fig. 14 Maximum-range solution without span constraint, including induced drag.

\section{B. Unconstrained Span}

The aerostructural optimization problem (5) is also solved with no span constraint. As before, two problems are compared: one considering only induced drag and another for which viscous drag is added.

\section{Induced Drag}

Since there is no constraint in the span, the only factor limiting the span is the tradeoff between the increase in weight due to an increase in bending moment and the reduction in induced drag.

Figure 14 shows the resulting optimum geometry, which exhibits segments with increasingly higher sweep toward the tip: i.e., a raked wingtip. Raked wingtips reduce the induced drag by interacting with the wing vortex wake, while providing a structural benefit by reducing the tip loads. As in the case of winglets, this effect becomes more pronounced at the critical maneuver condition, as can be seen in the lift distribution in Fig. 15a. Because the lift-curve slope decreases with sweep, the lift at the raked wingtip increases less rapidly than the other sections as the angle of attack is increased. This results in a spanwise lift distribution that is structurally more favorable at the higher angle of attack of the maneuver condition without sacrificing the cruise lift distribution significantly.

The projected semispan of the raked-wingtip configuration increases by $18 \%$ when compared to the span-constrained solution.
This increase in span results in a higher structural weight but also higher aerodynamic efficiency. The range of the raked-wingtip solution with an unconstrained span is $2 \%$ higher than the range of the optimal solution with the constrained span.

Another minimum was discovered by the optimizer: the winglet configuration whose lift distribution is shown in Fig. 15b. This configuration produces a more dramatic shift of the lift inboard, and the span is $9 \%$ smaller, which results in an overall reduction in structural weight of $5.1 \%$. The lift-to-drag ratio of the raked wingtip is $1 \%$ higher compared to the winglet configuration. A marginally better value for the maximum range $(0.5 \%$ higher $)$ is obtained for the raked-wingtip configuration. The loaded and unloaded twist distribution of the raked-wingtip configuration is shown in Fig. 16a. The raked segments show very high twist under increased load due to the high sweep angle of these segments. The thickness and stress distributions for the raked-wingtip configuration are shown in Figs. $16 \mathrm{~b}$ and $16 \mathrm{c}$, respectively. Under the maneuver loading condition, all elements are fully stressed except the tip element, which is at the minimum gauge thickness.

\section{Viscous and Compressibility Drag}

The same optimization problem is now analyzed with viscous effects included in the drag calculations. Figure 17 shows the optimal wing obtained with this optimization, which again exhibits a raked wingtip. The raked wingtip incorporates a significantly smaller portion of the overall span compared to the previous case. The wingtip is highly swept, with a sweep angle of $52^{\circ}$.

As with the induced-drag minimization problem, a local optimum in the form of a winglet configuration was found (see Fig. 18). The

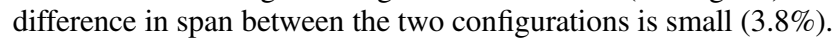

As in the previous cases for which viscous drag was considered, the root chords are larger than when only induced drag was considered. The root chords are 11 and $12 \%$ larger for the rakedwingtip and winglet configurations, respectively. This also allows for a larger projected span, since the diameter of the beam elements is determined from the local chord of the respective panels. The span of the raked-wingtip and winglet configurations are 6.2 and $9.3 \%$ larger than the respective cases for which only induced drag was considered.

The lift distributions for the raked-wingtip and winglet configurations are shown in Figs. 19a and 20a, respectively. They show a similar trend to the previous case. The winglet configuration experiences a higher loading toward the root of the main wing at the maneuver condition when compared to the raked-wingtip configuration. The difference between the critical maneuver lift distribution for the two configurations is small.

All beam elements are fully stressed, except for the tip element, whose stress is just under the material yield stress, as shown in Fig. 19d. The winglet beam element thicknesses are reduced to the minimum gauge thickness. The loaded and unloaded twist distributions for the two configurations are shown in Figs. 19b and

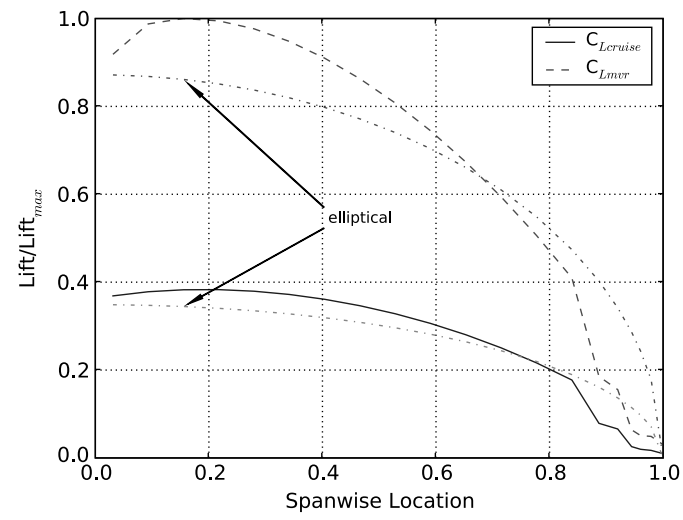

a) Raked-wingtip solution

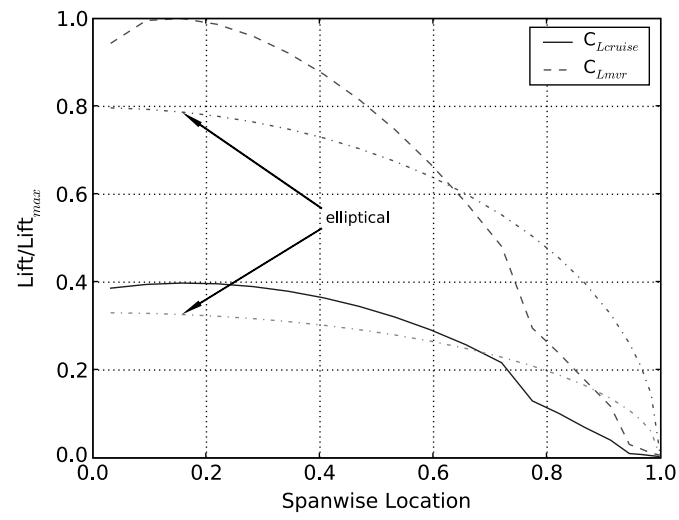

b) Winglet solution

Fig. 15 Spanwise lift distributions comparison for maximum-range solutions without span constraint, including induced drag. 


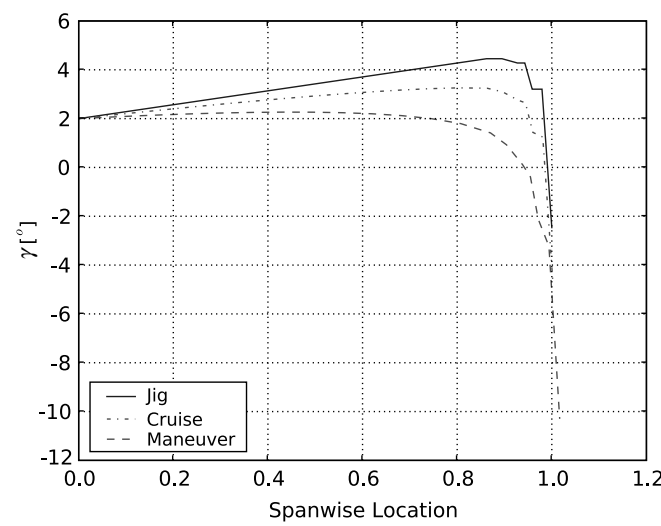

a) Twist distribution

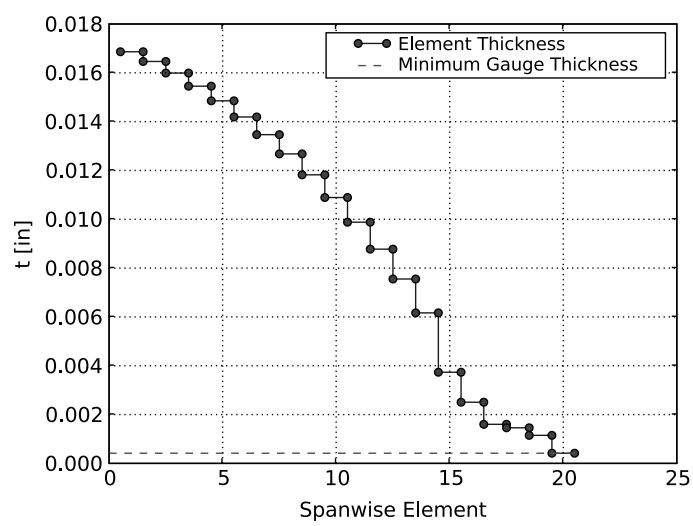

b) Thickness distribution

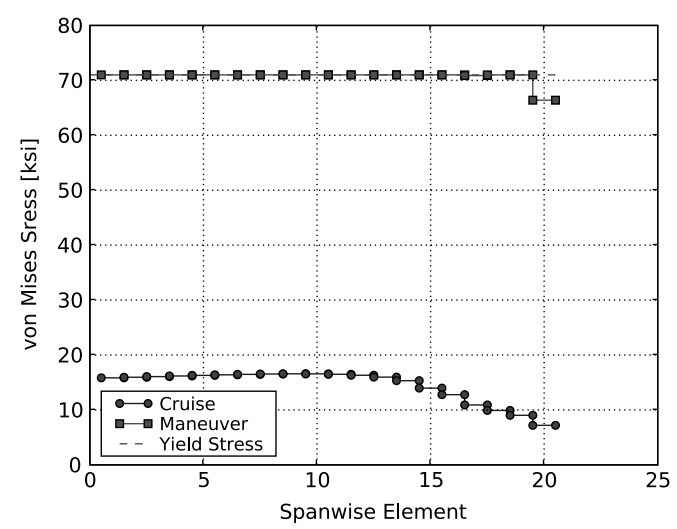

c) Stress distribution

Fig. 16 Spanwise distributions for maximum-range solution (raked wingtip) without span constraint, including induced drag.

$20 \mathrm{~b}$, respectively. The raked wingtip experiences high torsion compared to the winglet configuration, due to the high sweep. This results in a weight difference between the two configurations that is smaller than when viscous drag is not considered $(2.1 \%$ versus $5.1 \%$ ). The raked-wingtip configuration has a $2.2 \%$ higher lift-to-
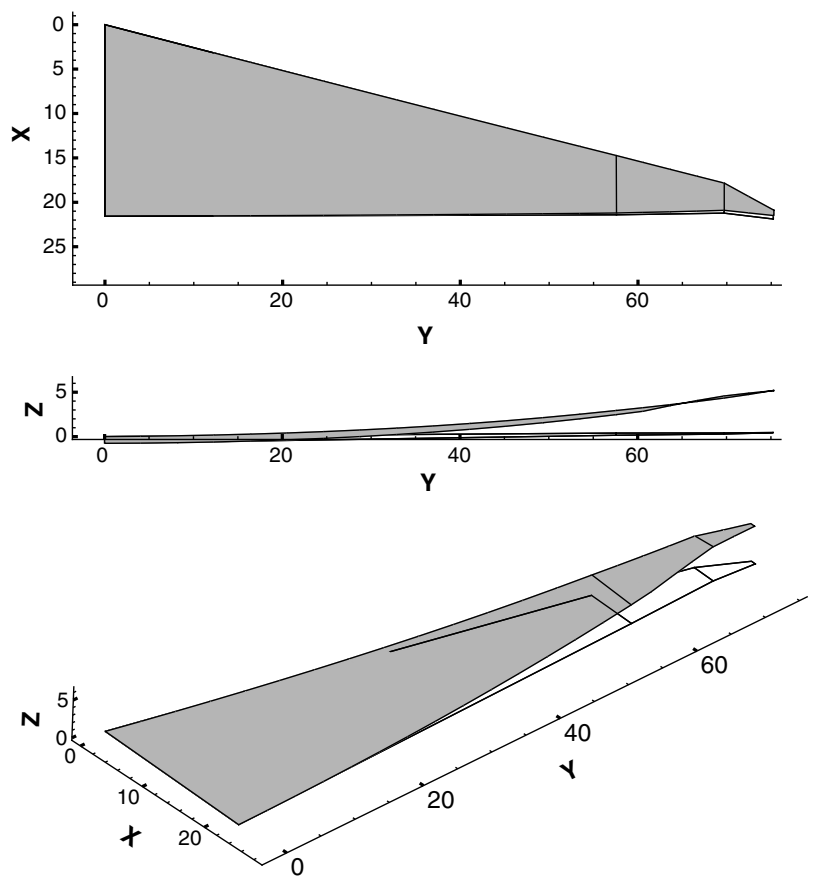

Fig. 17 Maximum-range solution (raked wingtip) without span constraint, including induced, compressibility, and viscous drag. drag ratio when compared to the winglet configuration. However, the raked-wingtip configuration results in a higher range.

The numerical results from the different optimization trials are summarized in Table 3. The structural weights listed in the table correspond to half of the wing and are based on the weight of the
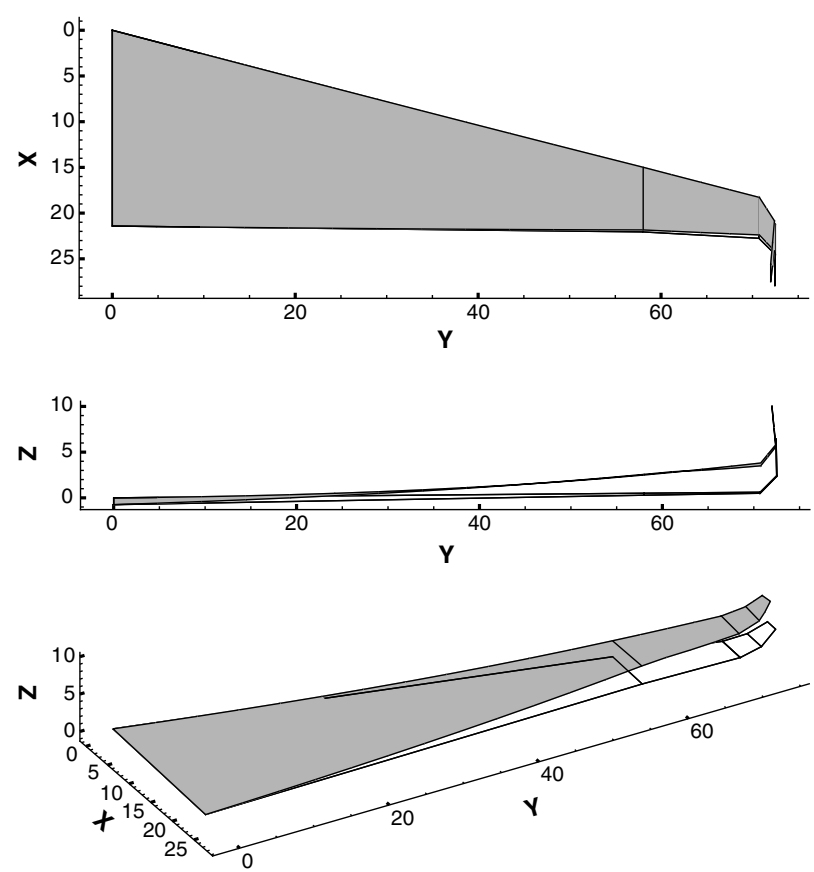

Fig. 18 Maximum-range solution (winglet) without span constraint, including induced, compressibility, and viscous drag. 


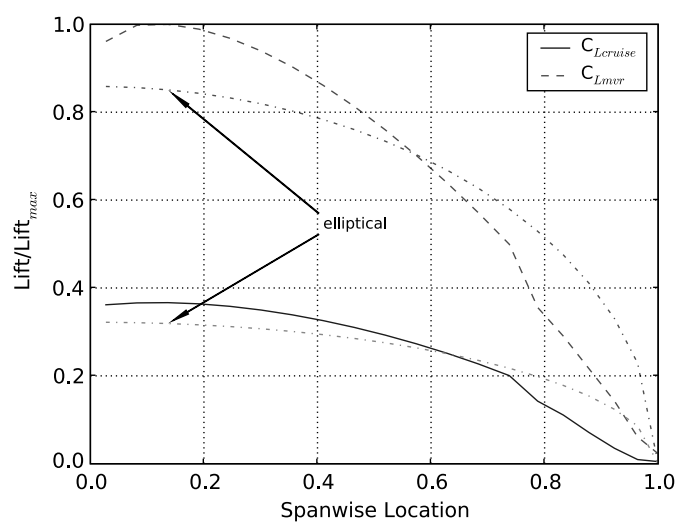

a) Lift distribution

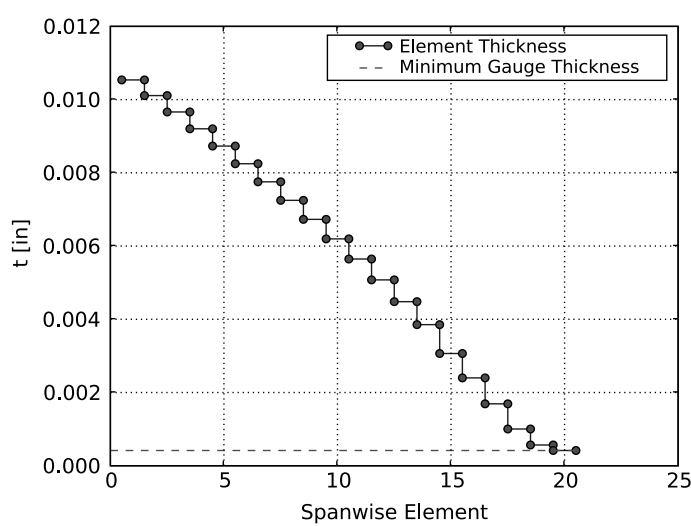

c) Thickness distribution

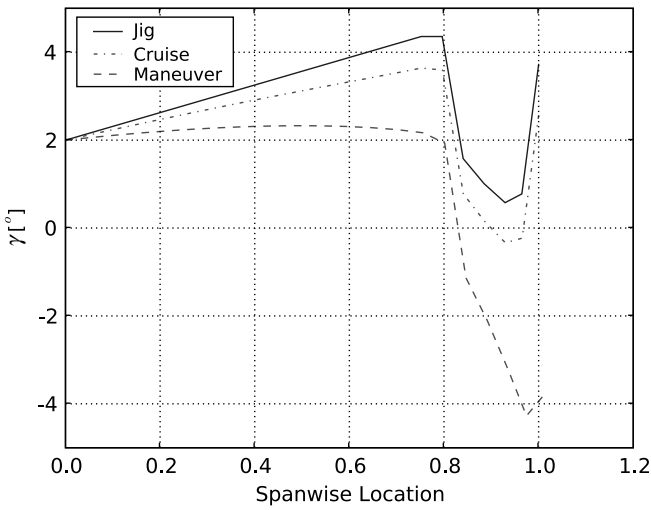

b) Twist distribution

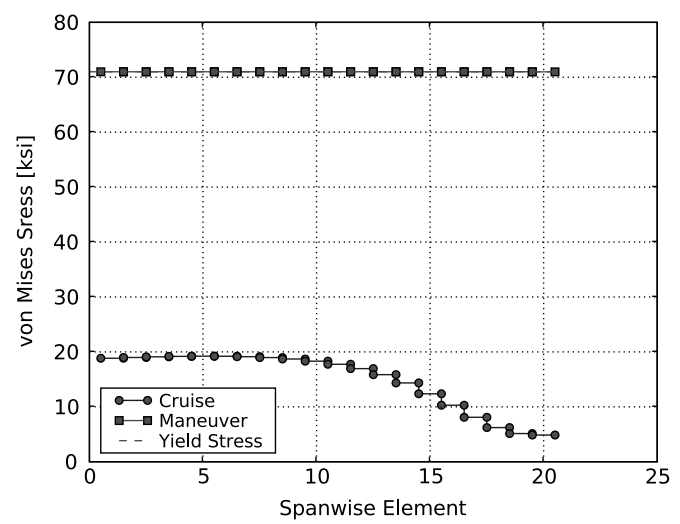

d) Stress distribution

Fig. 19 Spanwise distributions for maximum-range solution (raked wingtip) without span constraint, including induced, compressibility, and viscous drag.

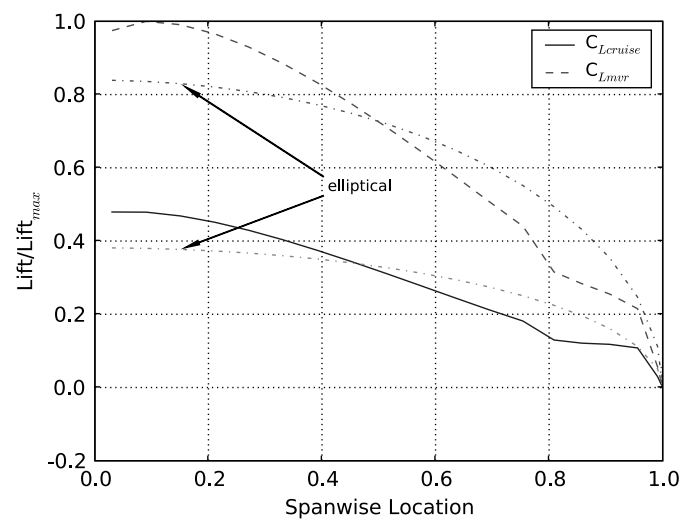

a) Lift distribution

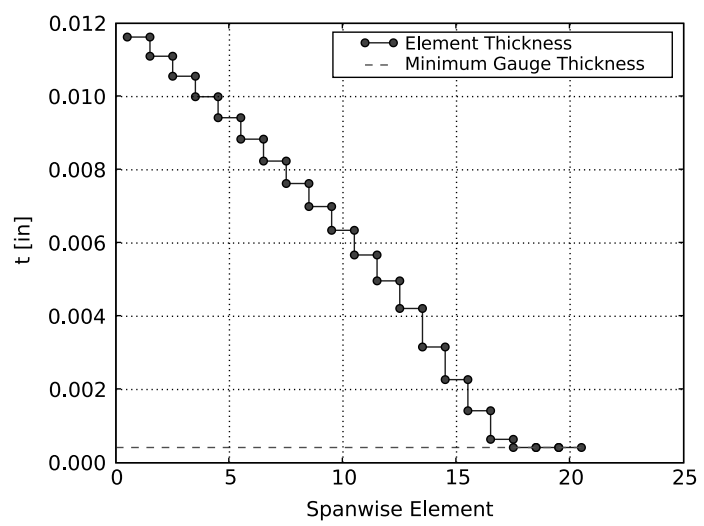

c) Thickness distribution

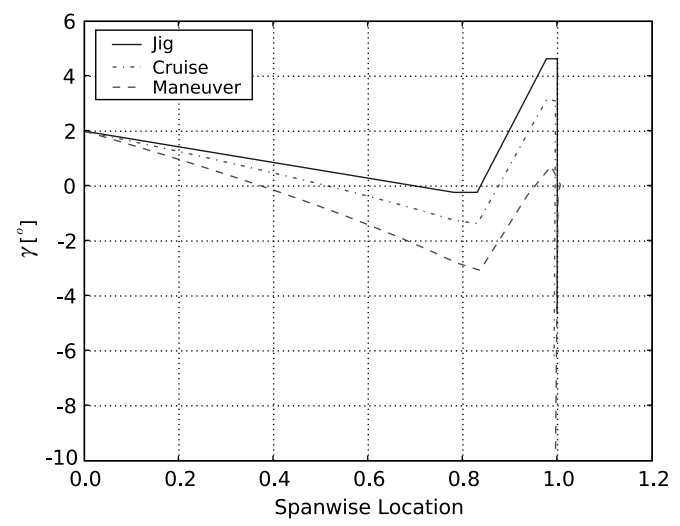

b) Twist distribution

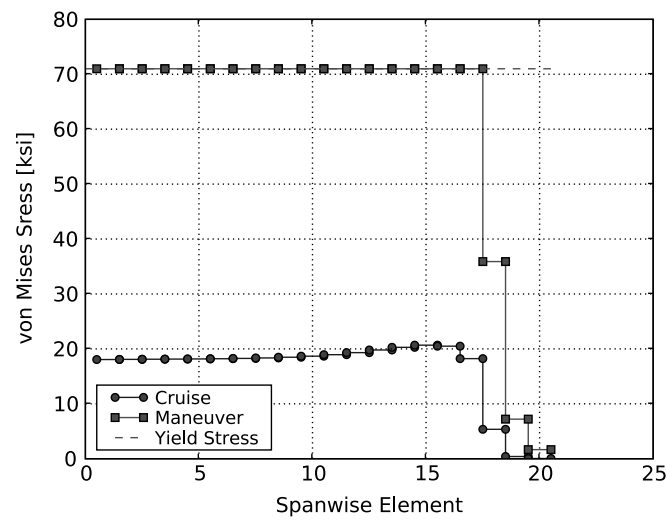

d) Stress distribution

Fig. 20 Spanwise distributions for maximum-range solution (winglet) without span constraint, including induced, compressibility, and viscous drag. 
Table 3 Aerostructural optimization results summary

\begin{tabular}{lccccc}
\hline \hline & Weight, $\mathrm{lb}$ & $L / D$ & Range, $\mathrm{nm}$ & $c_{r}, \mathrm{ft}$ & $b, \mathrm{ft}$ \\
\hline \multicolumn{7}{c}{ Span constrained with induced drag } \\
Winglet & 7,716 & 24.69 & 5,872 & 19.37 & 60.0 \\
Span constrained with induced and viscous drag & \\
Winglet & 6,389 & 17.74 & 4,243 & 21.41 & 60.0 \\
Winglet & Span unconstrained with induced drag \\
Raked wingtip & 9,795 & 26.41 & 5,936 & 19.14 & 66.31 \\
Span unconstrained with induced and viscous drag & \\
Winglet & 10,330 & 26.50 & 5,967 & 19.39 & 70.82 \\
Raked wingtip & 8,877 & 18.49 & 4,386 & 21.43 & 72.47 \\
\hline \hline
\end{tabular}

beam model multiplied by the correction factor that was previously explained.

\section{Conclusions}

In this study we found optimal nonplanar lifting-surface configurations by solving a series of wing design optimization problems. The problems differed in the disciplines that were considered (aerodynamics alone versus aerodynamics and structures), the enforcement of a span constraint, and the types of drag included in the aerodynamic model (starting with only induced drag, then adding compressibility drag and viscous drag).

For the aerodynamic optimization, box-wing or joined-wing configurations were found to be optimal when only induced drag was considered. When viscous drag was added, these configurations incurred a drag penalty due to the large surface area, and a C-wing configuration was preferred. In all cases, the nonplanar configurations provided a significant reduction in drag compared to the planar reference case. The reduction in drag was similar for all three cases, ranging from $26 \%$ for the joined wing to $22 \%$ for the $\mathrm{C}$-wing configuration.

The aerostructural model developed herein accounts for the interaction between the aerodynamics and the structure of the wing. The jig shape of the wing was designed while taking into account its flying shape and performance for both cruise and maneuver conditions. Aerodynamic shape and structural sizes were optimized, allowing the optimizer to perform the correct tradeoffs between the two disciplines. This was a significant improvement over previous approaches, where structural performance was considered by simply constraining the root bending moment.

The optimal solution for the aerostructural problem with a span constraint was a winglet configuration. The winglet allowed for a near-elliptical lift distribution at the cruise condition, while alleviating the tip loads by shifting lift distribution inboard at the critical maneuver condition and hence reducing material needed to satisfy the stress constraints. At the same time, the winglet provided an aerodynamic advantage by increasing the effective wingspan.

When the wingspan was not constrained, the optimal solution was a raked-wingtip extension. As in the case of the winglet configuration, the lift distribution shifted inboard at the maneuver condition, but to a lesser degree, resulting in a higher weight than the winglet configurations. At the same time, the aerodynamic performance of the extended wing provided a higher lift-to-drag ratio, resulting in a longer range for the raked-wingtip configuration. The difference in range between the winglet configuration and the rakedwingtip configuration was only $0.5 \%$ for the case in which only induced drag is considered. When viscous drag was included, the raked-wingtip configuration outperformed the winglet configuration by $2.2 \%$.

\section{References}

[1] Kroo, I., "Nonplanar Wing Concepts for Increased Aircraft Efficiency," Innovative Configurations and Advanced Concepts for Future Civil Aircraft, VKI Lecture Series, von Karman Inst., Rhode-Saint-Genèse, Belgium, June 6-10 2005.
[2] Hemke, P., "Drag of Wings With End Plates," NASA, Technical Report NASA TR 267, 1928.

[3] Mangler, W., "The Lift Distribution of Wings with End Plates," NASA TM 856, 1938.

[4] Whitcomb, R., "A Design Approach and Selected Wind Tunnel Results at High Subsonic Speeds for Wing-Tip Mounted Winglets," NASA TN D-8260, 1976

[5] Flechner, S., and Jacobs, P., "Experimental Results of Winglets on First, Second, and Third Generation Jet Transports," CTOL Transport Technology, Vol. 2, NASA CP-2036-PT-2, Hampton, VA, 1978, pp. 553-569.

[6] Jones, R., and Lasinski, T., "Effect of Winglets on the Induced Drag of Ideal Wing Shapes," NASA, TM 81230, 1980.

[7] Asai, K., "Theoretical Considerations in Aerodynamic Effectiveness of Winglets," Journal of Aircraft, Vol. 22, No. 7, July 1985, pp. 635-637. doi:10.2514/3.45177

[8] Downie, D., The Complete Guide to Rutan Aircraft, Tab Books, Blue Ridge Summit, PA, 1984.

[9] Terry, J., "Aerodynamic Characteristics of Ring Wings: A Bibliography," Redstone Scientific Information Center, Rept. RSIC285, Redstone Arsenal, AL, 1964.

[10] Miranda, L., "Boxplane Configuration for Conceptual Analysis and Initial Experimental Verification," Lockheed California Co., Rept. LR 25180, Burbank, CA, 1972.

[11] Wolkovitch, J., "The Joined Wing: An Overview," Journal of Aircraft, Vol. 23, 1986, pp. 161-178. doi: $10.2514 / 3.45285$

[12] Lee, H., Kim, Y., Park, G., Kolonay, R., Blair, M., and Canfield, R., "Structural Optimization of a Joined Wing Using Equivalent Static Loads," Journal of Aircraft, Vol. 44, No. 4, 2007, pp. 1302-1308. doi:10.2514/1.26869

[13] Hicken, J. E., "Efficient Algorithms for Future Aircraft Design: Contributions to Aerodynamic Shape Optimization," Ph.D. Thesis, Univ. of Toronto, Inst. for Aerospace Studies, Toronto, 2009.

[14] McMasters, J., and Kroo, I., "Advanced Configurations for Very Large Transport Airplanes," Aircraft Design, Vol. 1, 1998, pp. 217-242. doi:10.1016/S1369-8869(98)00018-4

[15] Gage, P., "New Approaches to Optimization in Aerospace Conceptual Design," Ph.D. Thesis, Stanford Univ., Stanford, CA, Dec. 1994.

[16] Verstraeten, J. G., and Slingerland, R., "Drag Characteristics for Optimally Span-Loaded Planar, Wingletted, and C-Wings," Journal of Aircraft, Vol. 46, No. 3, 2009, pp. 962-971. doi:10.2514/1.39426

[17] Ning, S. A., and Kroo, I., "Multidisciplinary Considerations in the Design of Wings and Wing Tip Devices," Journal of Aircraft, Vol. 47, No. 2, 2010, pp. 534-543. doi: $10.2514 / 1.41833$

[18] Martins, J., Alonso, J., and Reuther, J., "High-Fidelity Aerostructural Design Optimization of a Supersonic Business Jet," Journal of Aircraft, Vol. 41, No. 3, 2004, pp. 523-530. doi:10.2514/1.11478

[19] Johnson, F., Tinoco, E., and Yu, N., "Thirty Years of Development and Application of CFD at Boeing Commercial Airplanes, Seattle," AIAA Computational Fluid Dynamics Conference, AIAA Paper 2003-3439, Orlando, FL, June 23-26 2003.

[20] Katz, J., and Plotkin, A., Low-Speed Aerodynamics, 2nd ed., Cambridge Univ. Press, Cambridge, England, U.K., 2001.

[21] Liersch, C., and Wunderlich, T., "A Fast Aerodynamic Tool for Preliminary Aircraft Design," 12th AIAA/ISSMO Multidisciplinary Analysis and Optimization Conference, AIAA Paper 2000-3901, Victoria, BC, Canada, Sept. 2008.

[22] Sivellis, C., "Experimental and Calculated Characteristics of Three Wings of NACA 64-210 and 65-210 Airfoil Sections," NACATN 1422, 1947.

[23] Hajela, P., "Preliminary Weight Estimation of Conventional and Joined Wings Using Equivalent Beam Models," Journal of Aircraft, Vol. 25, No. 6, 1988, pp. 574-576. doi: $10.2514 / 3.45625$

[24] Kuttenkeuler, J., and Ringertz, U., "Aeroelastic Design Optimization with Experimental Verification," Journal of Aircraft, Vol. 35, No. 3, 1998 , pp. 505-507. doi: $10.2514 / 2.2330$

[25] Przemieniecki, J., Theory of Matrix Structural Analysis, Dover, New York, 1985.

[26] Martins, J. R. R. A., Alonso, J. J., and Reuther, J. J., "A Coupled-Adjoint Sensitivity Analysis Method for High-Fidelity Aero-Structural Design," Optimization and Engineering, Vol. 6, No. 1, March 2005, pp. 33-62. doi:10.1023/B:OPTE.0000048536.47956.62 
[27] Perez, R. E., and Behdinan, K., "Particle Swarm Optimization in Structural Design, Swarm Intelligence: Focus on Ant and Particle Swarm Optimization, 1st ed., I-Tech Education and Publishing, Vienna, Dec. 2007, pp. 373-394.

[28] Eberhart, R., and Kennedy, J., "New Optimizer Using Particle Swarm Theory," Sixth International Symposium on Micro Machine and Human Science, Nagoya, Japan, 1995, pp. 39-43.

[29] Kennedy, J., and Eberhart, R., "Particle Swarm Optimization," IEEE International Conference on Neural Networks, Vol. 4, Inst. of Electrical and Electronics Engineers, Piscataway, NJ, 1995, pp. 1942-1948.

[30] Hu, X., Eberhart, R., and Shi, Y., "Engineering Optimization with Particle Swarm," IEEE Swarm Intelligence Symposium 2003 (SIS 2003), Inst. of Electrical and Electronics Engineers, Piscataway, NJ, 2003, pp. 53-57.

[31] Perez, R. E., and Behdinan, K., "Particle Swarm Approach for Structural Design," Computers and Structures, Vol. 85, Oct. 2007, pp. $1579-1588$.

doi:10.1016/j.compstruc.2006.10.013
[32] Hassan, R., Cohanim, B., de Week, O., and Venter, C., "A Comparison of Particle Swarm Optimization and the Genetic Algorithm," 1st AIAA Multidisciplinary Design Optimization Specialist Conference, AIAA Paper 2005-1897, Austin, TX, April 2005.

[33] Gill, P., Murray, W., and Saunders, M., "SNOPT: An SQPAlgorithm for Large-Scale Constraint Optimization," SIAM Journal on Optimization, Vol. 12, No. 4, 2002, pp. 979-1006. doi:10.1137/S1052623499350013

[34] Perez, R. E., Jansen, P. W., and Martins, J. R. R. A., "Aerostructural Optimization of Non-Planar Lifting Surfaces," 12th AIAA/ISSMO Multidisciplinary Analysis and Optimization Conference, Victoria, BC, Canada, AIAA Paper 2008-5967, Sept. 2008.

[35] "Airworthiness Standards: Transport Category Airplanes," Federal Aviation Administration, Federal Aviation Regulations, Part 25, Feb. 2009.

[36] Cramer, E. J., Dennis, J. E., Frank, P. D., Lewis, R. M., and Shubin, G. R., "Problem Formulation for Multidisciplinary Optimization," SIAM Journal on Optimization, Vol. 4, No. 4, 1994, pp. 754-776. doi: $10.1137 / 0804044$ 